\title{
BMJ Open Effect of Baduanjin exercise on cognitive function in older adults with mild cognitive impairment: study protocol for a randomised controlled trial
}

\author{
Guohua Zheng, ${ }^{1}$ Maomao Huang, ${ }^{1}$ Shuzhen Li, ${ }^{1}$ Moyi Li, ${ }^{1}$ Rui Xia, ${ }^{1}$ Wenji Zhou, ${ }^{1}$ \\ Jing Tao, ${ }^{1,2}$ Lidian Chen ${ }^{3}$
}

To cite: Zheng G, Huang M, Li S, et al. Effect of Baduanjin exercise on cognitive function in older adults with mild cognitive impairment: study protocol for a randomised controlled trial. BMJ Open 2016;6:e010602 doi:10.1136/bmjopen-2015010602

- Prepublication history for this paper is available online. To view these files please visit the journal online (http://dx.doi.org/10.1136/ bmjopen-2015-010602).

GZ and MH contributed equally.

Received 19 November 2015 Revised 29 January 2016 Accepted 2 March 2016

CrossMark

${ }^{1}$ College of Rehabilitation Medicine, Fujian University of Traditional Chinese Medicine, Fuzhou, Fujian, China

${ }^{2}$ Fujian Key Laboratory of Rehabilitation Technology and Rehabilitation Research Center for Traditional Chinese Medicine, Fuzhou, Fujian, China

${ }^{3}$ Fujian University of

Traditional Chinese Medicine, Fuzhou, Fujian, China

Correspondence to Professor Lidian Chen; cld@fjtcm.edu.cn

\section{ABSTRACT}

Introduction: Mild cognitive impairment (MCl) is an intermediate stage between the cognitive changes of normal aging and dementia characterised by a reduction in memory and/or other cognitive processes. An increasing number of studies have indicated that regular physical activity/exercise may have beneficial association with cognitive function of older adults with or without cognitive impairment. As a traditional Chinese Qigong exercise, Baduanjin may be even more beneficial in promoting cognitive ability in older adults with $\mathrm{MCl}$, but the evidence is still insufficient. The main purpose of this study is to investigate the effect of Baduanjin exercise on neuropsychological outcomes of community-dwelling older adults with $\mathrm{MCl}$, and to explore its mechanism of action from neuroimaging based on functional MRI (fMRI) and cerebrovascular function.

Methods and analysis: The design of this study is a randomised, controlled trial with three parallel groups in a 1:1:1 allocation ratio with allocation concealment and assessor blinding. A total of 135 participants will be enrolled and randomised to the 24-week Baduanjin exercise intervention, 24-week brisk walking intervention and 24-week usual physical activity control group. Global cognitive function and the specific domains of cognition (memory, processing speed, executive function, attention and verbal learning and memory) will be assessed at baseline and 9, 17, 25 and 37 weeks after randomisation, while the structure and function of brain regions related to cognitive function and haemodynamic variables of the brain will be measured by fMRI and transcranial Doppler, respectively, at baseline and 25 and 37 weeks after randomisation.

Ethics and dissemination: Ethics approval was given by the Medical Ethics Committee of the Second People's Hospital of Fujian Province (approval number 2014-KL045-02). The findings will be disseminated through peer-reviewed publications and at scientific conferences.

Trial registration number: ChiCTR-ICR-15005795; Pre-results.
Strengths and limitations of this study

- This study will evaluate the effects of Baduanjin exercise on cognitive ability of older adults with mild cognitive impairment (MCl) and explore whether this effect is superior to the brisk walking intervention.

- A prospective, randomised, controlled, three-arm study with blinded outcome measurements will be performed.

- A broad measurement tool and multiple measurement time points will be used to judge the effects of interventions (Baduanjin or brisk walking) on cognitive ability.

- The efficacy of a 24-week Baduanjin exercise intervention in older adults with $\mathrm{MCl}$ in improving cognitive ability remains to be determined.

\section{BACKGROUND}

Nowadays, nearly 47 million people are living with dementia worldwide, with about 10 million new cases being diagnosed every year; this number is projected to nearly triple to more than 130 million by 2050 year. ${ }^{1}$ Patients with dementia usually have neuropsychiatric symptoms and a progressive decline in their $\operatorname{cog}$ nition and functional ability. ${ }^{23}$ These mental disorders seriously affect their quality of life and capacity for daily activity. ${ }^{4}$ However, there is as yet no really satisfactory treatment to block the progress of dementia. Mild $\operatorname{cog}$ nitive impairment (MCI) is an intermediate stage between the cognitive changes of normal aging and dementia characterised by a reduction in memory and/or other cognitive processes. ${ }^{5}$ Older adults with MCI are at increased risk of conversion to dementia or clinical Alzheimer's disease (AD), with progression rates of $10-20 \%$ per year compared with $1-2 \%$ among cognitively normal people 
at the same age. ${ }^{6}$ What's more, MCI contributes to decreased quality of life, increased neuropsychiatric symptoms and disability, ${ }^{7}$ as well as increased healthcare costs. As a possible prelude to dementia or $\mathrm{AD}, \mathrm{MCI}$ has become an important public health problem throughout the world. ${ }^{8}$ However, as a heterogeneous entity characterised by differences in cognitive profile, the outcome of MCI is variable and uncertain. ${ }^{9}$ Many patients with MCI remain stable or even revert to a normal state if appropriate management is adopted. In population-based studies, rates of 'reversion to normal' among elderly with MCI have been found to reach 14$40 \%$ within 2-3 years. ${ }^{10}{ }^{11}$ Furthermore, emerging evidence shows that early improvement in MCI is very beneficial for decreasing the prevalence of dementia or $\mathrm{AD} .{ }^{12}$ Thus, there is an urgent need to find effective approaches to treating MCI.

An increasing number of studies have indicated that physical activity/exercise may have beneficial effects associated with cognitive function of older adults with or without cognitive impairment. Epidemiological research shows an inverse relationship between levels of physical activity/exercise (especially aerobic exercise) and risk of cognitive decline and dementia in older adults with or without cognitive impairment. ${ }^{13-15}$ This evidence is supported by cross-sectional studies, longitudinal observational studies and prospective intervention trials. ${ }^{16-19}$ The mechanism is still uncertain, but exercise may improve cerebral circulation by increasing blood flow to the brain and changing the structure and function of brain tissue by increasing angiogenesis, neurogenesis, synaptogenesis and the synthesis of neurotransmitters. ${ }^{20}{ }^{21}$ However, recent systematic reviews call for caution when interpreting the relationship between exercise and cognitive function in older adults with MCI because of variability in study design, population characteristics and exercise parameters. ${ }^{22}{ }^{23}$ Exercise type and intensity differ and some interventions may not be long enough to observe changes in cognitive function, and exercise durations of at least 6 months are often needed before cognitive changes are detected. ${ }^{24} 25$ Therefore, type and intensity designed to facilitate longterm exercise participation should be incorporated into the design of exercise programmes for older adults.

An important component of traditional Chinese Qigong exercises, Baduanjin exercise is an ancient art and science of healthcare that has been practised in China for more than 1000 years. ${ }^{26}$ It is a safe aerobic exercise based on the common rules of Qigong exercise, combined with the holistic view and the theory of Qi in traditional Chinese medicine. ${ }^{27}$ However, it is different from other types of aerobic exercise in that practitioners are required to reach coordination between mind and body. ${ }^{28}$ Studies have demonstrated that regular practice of Baduanjin exercise can result in physiological benefits related to cardiopulmonary function, ${ }^{29}$ balance, ${ }^{30}$ autonomic nerves, ${ }^{31}$ osteoarthritis ${ }^{32}$ and bone loss prevention $^{33}$ in older people, and is therefore recommended for older adults. To date, however, there have been no trials evaluating the effect of Baduanjin on cognitive function for older adults with or without cognitive impairment, but some studies have shown that regular Baduanjin training can slow normal age-related decline in the memory domain, delay aging of intelligence in older adults, ${ }^{34}$ and have a better effect than jogging. ${ }^{35}$ In addition, some studies report that Baduanjin exercise has a positive effect in lowering blood pressure ${ }^{36}$ blood lipid $^{37}$ and inflammatory factors, ${ }^{38}$ which are risk factors for cognitive impairment, and improving quality of life, fatigue $^{39}$ and mood status ${ }^{40}$ of older adults, and these variables are also associated with self-reported cognitive symptoms. ${ }^{41}$ As a result, it is reasonable to assume that regular Baduanjin training could be beneficial to cognitive function in older adults with or without cognitive impairment, and it is more effective than usual aerobic exercise. The main purpose of this study is to conduct a methodologically rigorous randomised controlled trial (RCT) to systematically investigate the effects of Baduanjin exercise on neuropsychological outcomes in community-dwelling older adults with MCI and its possible mechanism, and compare it with a usual aerobic exercise (brisk walking).

\section{METHOD/DESIGN}

\section{Study aims and hypotheses}

The primary aim of this study is to assess the effect of Baduanjin exercise on cognitive function in older adults with MCI.

The primary hypothesis of this study is that older adults with MCI who receive a 24-week Baduanjin training intervention will show greater improvement in global cognitive ability than those who keep to their usual physical activities, as assessed immediately after the 24-week Baduanjin exercise intervention, and these benefits will continue until the end of a 12-week follow-up.

The secondary aims of this study are:

1. To investigate if Baduanjin is more beneficial to $\operatorname{cog}$ nitive function of older adults with MCI than the usual aerobic exercise of brisk walking

2. To investigate the effect of Baduanjin on specific domains of cognition in older adults with MCI

3. To explore the mechanism of action of Baduanjin on cognitive function in older adults with MCI based on functional MRI (fMRI) and cerebrovascular function.

We hypothesise that older adults with MCI who receive a 24-week Baduanjin training intervention will achieve greater improvement in specific domains of cognition and cerebral haemodynamics and show favourable changes in structure and function of related brain regions than those who maintain their usual physical activities or even the brisk walking intervention.

\section{Study design}

The design of this study is an RCT with three parallel groups in a 1:1:1 allocation ratio with allocation concealment and assessor blinding. The design follows the Standard Protocol Items Recommendations for 
Interventional Trials (SPIRIT) ${ }^{42}$ and the Consolidated Standards of Reporting Trials (CONSORT) ${ }^{43}$ A total of 135 eligible participants will be enrolled from the Cangxia Community Center in Taijiang District and Longfeng Community Center in Gulou District, Fuzhou City, China, and will be randomised to the 24-week Baduanjin exercise intervention (60 min per session, three sessions per week), 24-week brisk walking intervention (60 min per session, three sessions per week) or the 24-week usual activity control group. Global cognitive function and specific domains of cognition (memory, processing speed, executive function, attention and verbal learning and memory) will be assessed at baseline before randomisation and at 9, 17, 25 (at the end of intervention) and 37 weeks (after the 12-week follow-up period), while the structure and function of brain regions related to cognitive function and haemodynamic variables will be measured using fMRI and transcranial Doppler (TCD), respectively, at baseline before randomisation and at 25 and 37 weeks. All data collectors will be blinded to the group assignments. The flow diagram for this trial is presented in figure 1.

\section{Study population}

The study population is older adults with a diagnosis of MCI living in the community in Fuzhou City. The diagnostic, inclusion and exclusion criteria for the study sample are as follows.

\section{Diagnostic criteria}

According to the Peterson diagnostic criteria of MCI, ${ }^{44}$ the participants with MCI should have: (1) memory problems, preferably confirmed by an informant; (2) cognitive decline in accordance with age and education; (3) intact activities of daily living (ADL) (Lawton-Brody ADL score $<18$ ); (4) absence of dementia (Global Deterioration Scale (GDS) score at 2 or 3 level).

\section{Inclusion criteria}

The eligible participants must meet the following criteria: (1) diagnosis of MCI; (2) aged 60 years or older; (3) no regular physical exercise for at least half a year (regular exercise means exercise with a frequency of at least twice a week and at least 20 min per session); (4) informed consent.

\section{Exclusion criteria}

Exclusion criteria include: (1) resistant hypertension; (2) severe visual or hearing loss; (3) evidence of severe psychiatric conditions (eg, severe depression, active suicidal ideation, schizophrenia); (4) current severe medical conditions for which exercise is contraindicated; (5) history of alcohol and drug abuse; (6) participating in another clinical study.

\section{Withdrawal criteria and management}

Participants will be allowed, or required, to withdraw from the trial based on the following: (1) a major protocol violation; (2) development of a serious disease preventing continuation in the trial; (3) adverse events related to the Baduanjin or brisk walking exercise; (4) request to be withdrawn from the trial.

\section{Recruitment and screening}

Participants will be recruited from the Cangxia Community Center of Taijiang District and the Longfeng Community Center of Gulou District in Fuzhou City by posting posters, sending leaflets and brochures, and setting up a recruiting station at the corresponding community centre. Potential participants will first complete a screening questionnaire and assessment of cognitive function to determine their eligibility. Eligible participants will receive information about this trial and have an informed discussion with trained research assistants regarding the information provided. Those who are interested in participating will have the baseline assessment of outcomes arranged. Written informed consent will be obtained by research assistants before the baseline assessment.

\section{Randomisation, allocation concealment, and blinding}

After baseline assessment, the eligible participants will be randomly allocated with equal rate to their respective groups. The random allocation sequence will be produced using the PLAN procedure of the statistical software SAS v.9.1 and be managed by an independent statistician blinded to the recruitment, evaluation and intervention of the participants. The research assistants will request (via telephone) a participant's allocation from the statistician. We cannot mask participants, exercise coaches, or intervention supervisors to the allocated treatment, but outcome assessors and data statisticians will be blinded to group allocation.

\section{Intervention}

\section{Baduanjin exercise group}

Participants in the Baduanjin exercise group will receive 24 weeks of Baduanjin exercise training with a frequency of 3 days a week and $60 \mathrm{~min}$ a day including $15 \mathrm{~min}$ warm up, $40 \mathrm{~min}$ Baduanjin training and $5 \mathrm{~min}$ cool down, and health education about cognition once every 8 weeks (30 min per session). Baduanjin training will be assigned at two community centres (Cangxia Community Center of Taijian District and Longfeng Community Center of Gulou District in Fuzhou City) with 20-25 individuals per centre. Two professional coaches, who have been engaged in teaching Baduanjin exercise to college students at the Fujian University of Traditional Chinese Medicine (FJTCM) for at least 5 years, will be employed to guide participants' training. The training scheme of Baduanjin exercise is planned according to the 'Health Qigong Baduanjin Standard' enacted by the State Sports General Administration in 2003, ${ }^{45}$ and consists of 10 postures (including the preparation and ending posture) (figure 2). ${ }^{46}$ A Polar Heart Rate Monitor (Mio Sport SD) will be used to monitor participants' energy consumption and heart rate (HR) during training. 


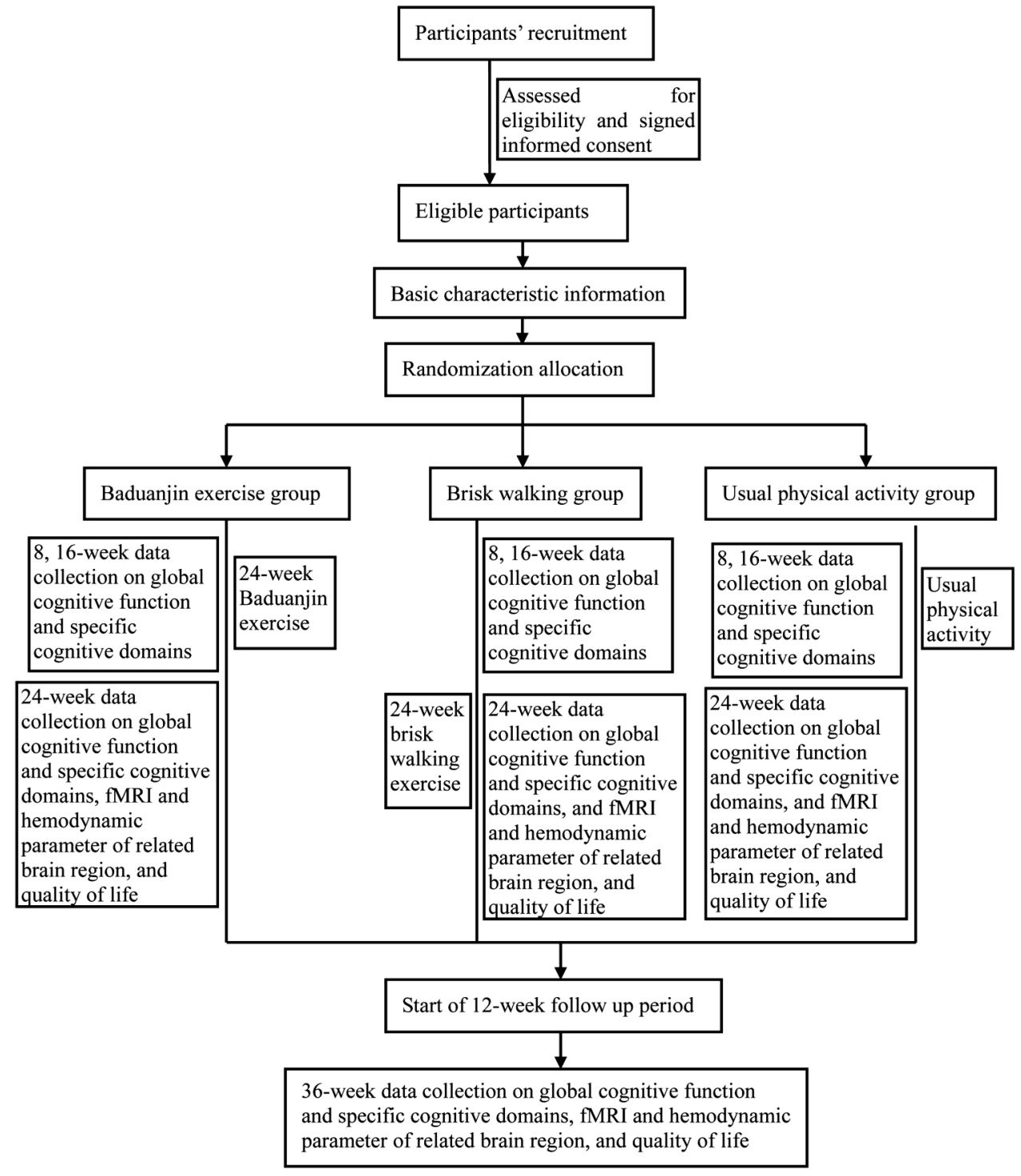

Figure 1 Flow diagram of study design.

\section{Brisk walking group}

Besides receiving health education like the Baduanjin exercise group, participants in the brisk walking group will receive 24 weeks of brisk walking training at Cangxia Community Center or Longfeng Community Center with a frequency of 3 days a week and 60 min a day including $15 \mathrm{~min}$ warm up, $40 \mathrm{~min}$ walking and $5 \mathrm{~min}$ cool down. Professional coaches will be employed to guide participants' training. The intensity of exercise will be controlled by monitoring participants' HR at $55-75 \%$ of their HR reserve through the Polar Heart Rate Monitor. ${ }^{47}$

\section{Usual physical activity group}

Participants in the usual physical activity group will not receive any specific exercise intervention besides health education with a frequency of one session every 8 weeks (30 min per session) like the training groups. They will maintain their original physical activity habits.

All participants will be advised not to seek any other regular exercise during the trial period. Two research assistants will supervise the training area to guarantee the quality of Baduanjin or brisk walking exercise. In order to observe any impact of the usual physical activity, we will require all participants to record information about their usual daily physical activity at 1, 13 and 24 weeks of the intervention period on a daily activity log.

\section{Follow-up}

After the 24-week intervention period, all participants will start an additional 12-week unsupervised follow-up period. During the follow-up period, all participants will be required to record their daily activity information at 4,8 and 12 weeks.

\section{Outcome assessment}

The variables in this trial consist of basic characteristics, primary outcomes and secondary outcomes. The basic characteristics will be measured at baseline (1-2 weeks before randomised allocation). Primary and secondary outcomes will be measured at baseline, end of the 


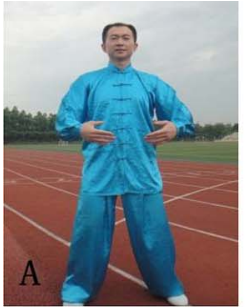

A: Preparation posture

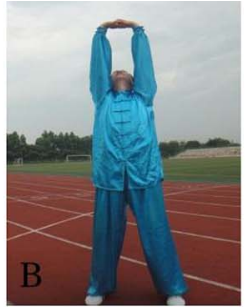

B: Prop up the sky by two hands to regulate tri-jiao

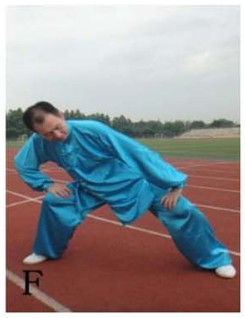

F: Shake head and buttocks to expel Heart (Xin)-fire

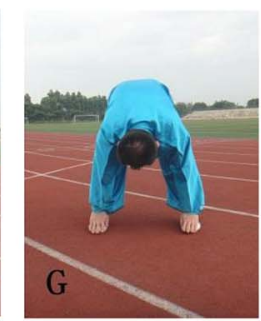

G: Pull toes with both hands to reinforce the kidney(shen) and waist

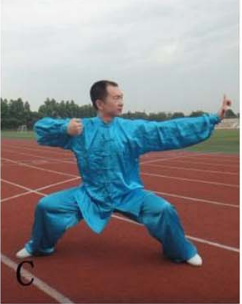

C: Draw a bow on both sides like shooting a vulture

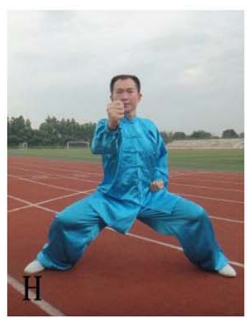

$\mathrm{H}$ : Clench one's fist and glare to increase strength

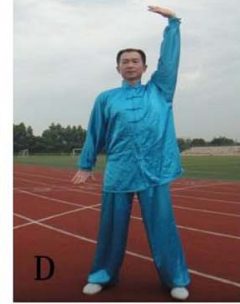

D: Raise single arm up to regulate spleen (Pi) and stomach (Wei)

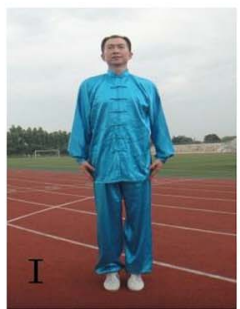

I: Rise and fall on tiptoe seven times to treat all diseases

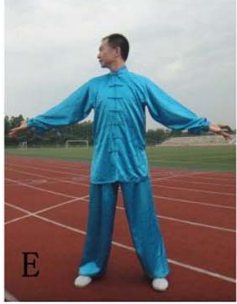

E: Look back to treat five strains and seven impairments

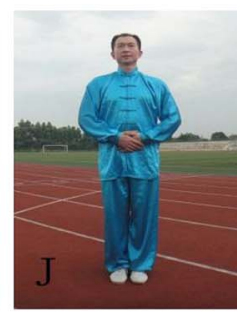

J: Ending posture intervention period (25 weeks after randomisation), and end of the follow-up period ( 37 weeks after randomisation). In addition, the outcomes of cognitive function including global cognitive function and specific cognitive domains (ie, memory, processing speed, executive function, attention and verbal learning and memory) will be repeatedly assessed every 2 months during the intervention period. All primary and secondary outcomes will be assessed by the experienced staff at the Affiliated Rehabilitation Hospital of FJTCM, who will be blinded to the allocation results of participants. A summary of all measurements in this trial is shown in table 1.

\section{Basic characteristics}

Participants' demographic characteristics (eg, age, sex, education, marital status, living arrangements, occupation and socioeconomic status) and history of disease and medication use will be collected by the recruiters using the self-designed questionnaire. The basic Montreal Cognitive Assessment (MoCA) score, GDS score, ADL scores and depression will be assessed using the corresponding standardised scales. Baseline assessment will be completed before randomisation.

\section{Primary outcomes}

Global cognitive function will be measured using the MoCA scale, which is a cognitive screening instrument created and validated to detect MCI. ${ }^{48}$ MoCA is a brief (about $10 \mathrm{~min}$ ) test that evaluates visuospatial/executive functions, naming, verbal memory registration and learning, attention, abstraction, 5 min delayed verbal memory, and orientation with a total score of 0-30 (a higher score equals better function). The Chinese version MoCA (Beijing version) was revised by Wang Wei and Jie Hengge in 2006, ${ }^{49}$ and is widely used in China with good validity, reliability and sensitivity. ${ }^{50}$

\section{Secondary outcomes}

Executive function will be measured using the Trail Making Test (TMT) and the Color-Word Matching Stroop task. The TMT consists of two parts (TMT-A and TMT-B): TMT-A requires individuals to sequentially connect 25 encircled numbers on a sheet of paper, while TMT-B requires participants to draw a line alternating between numbers and letters, both of which must be connected in ascending order. ${ }^{51}$ TMT-A places few cognitive demands on participants and primarily captures processing speed; while TMT-B requires more complex attention shifting, planning and concentration, and the Trails $\mathrm{B} / \mathrm{A}$ ratio is taken as a valid index of executive function. ${ }^{52}$ The Color-Word Matching Stroop task (cwmStroop) also assesses executive function. Two rows of letters appear on the screen, and subjects are requested to press a button, either A (Yes response) or $\mathrm{L}$ (No response) on the keyboard, as quickly as possible to 
Table 1 Outline and timelines of the assessments

\begin{tabular}{|c|c|c|c|c|c|}
\hline \multirow[b]{2}{*}{ Item } & \multirow{2}{*}{$\begin{array}{l}\text { Before } \\
\text { randomisation } \\
-2 \text { to }-1 \text { week }\end{array}$} & \multicolumn{2}{|c|}{$\begin{array}{l}\text { Intervention period } \\
\text { (1-24 weeks after } \\
\text { randomisation) }\end{array}$} & \multirow{2}{*}{$\begin{array}{l}\text { Follow-up period } \\
\text { (25-36 weeks after } \\
\text { randomisation) } \\
25 \text { weeks }\end{array}$} & \multirow{2}{*}{$\begin{array}{l}\text { After } \\
\text { randomisation } \\
37 \text { weeks }\end{array}$} \\
\hline & & 9 weeks & 17 weeks & & \\
\hline \multicolumn{6}{|l|}{ Eligible assessment } \\
\hline Diagnostic criteria & $x$ & & & & \\
\hline Inclusion criteria & $x$ & & & & \\
\hline Exclusion criteria & $x$ & & & & \\
\hline Baseline measurement & $x$ & & & & \\
\hline \multicolumn{6}{|l|}{ Primary outcomes } \\
\hline Global cognitive function & $x$ & $x$ & $x$ & $x$ & $x$ \\
\hline \multicolumn{6}{|l|}{ Secondary outcomes } \\
\hline Memory & $x$ & $\times$ & $x$ & $\times$ & $x$ \\
\hline Attention & $x$ & $x$ & $x$ & $x$ & $x$ \\
\hline Processing speed & $x$ & $x$ & $x$ & $x$ & $x$ \\
\hline Execution & $x$ & $x$ & $x$ & $x$ & $x$ \\
\hline Verbal learning and memory & $x$ & $x$ & $x$ & $x$ & $x$ \\
\hline $\begin{array}{l}\text { Structure and function of related } \\
\text { brain regions }\end{array}$ & $x$ & & & $x$ & $x$ \\
\hline Cerebrovascular function & $x$ & & & $\times$ & $x$ \\
\hline Quality of life & $x$ & & & $\times$ & $x$ \\
\hline Adverse events & & & & & $x$ \\
\hline
\end{tabular}

decide whether the colour name of the top row letters corresponds to the colour name written in black ink in the bottom row. ${ }^{53}$ The relative response time (RT) (ie, RT in the incongruent condition minus RT in the neutral condition) reflects executive ability. ${ }^{54}$

Memory will be evaluated using the Chinese Wechsler Memory Scale Revised (WMS-RC) and the Rey Auditory Verbal Learning Test (AVLT). WMS-RC is one of the most important tests of memory in China. ${ }^{55}$ This test consists of 10 parts: personal and current information, orientation, digital sequence relationship, picture recall, visual recognition, visual reproduction, associate learning, tactual performance test, logical memory and digit span, ${ }^{56}$ which can assess long-term, short-term and immediate memory. The score of the WMS-RC is expressed as a memory quotient (MQ), and higher scores of $\mathrm{MQ}$ equal better memory. AVLT is a commonly used neuropsychological measure that assesses verbal learning and memory including three subtests: immediate recall, short-term-delayed recall and long-delayed recognition. ${ }^{57}$ In the immediate recall test, the assessor says a list of 15 unrelated words three times, and participants are asked to repeat it immediately; the score of the immediate recall test is the sum of three-time scores. After $15 \mathrm{~min}$, participants are asked to repeat the 15 words, and the score of short-term-delayed recall is the number of words recalled correctly. In the long-delayed recognition test, participants are given another list of 15 unrelated words and must recognise the original list of 15 words. ${ }^{58}$ The Chinese version of AVLT has been reported by Guo Qi-hao and colleagues, and has reliable split-half reliability, internal consistency, and criterion and structure validity. ${ }^{59}$

Processing speed will be evaluated by digit-symbol coding (DSC) which is a subset of the Wechsler Adult Intelligence Scale-Revised China (WAIS-RC) ${ }^{60}$ DSC is a brief test that has nine numbers from 1 to 9 with nine corresponding symbols. Participants are required to draw each symbol under its corresponding number within a $90 \mathrm{~s}$ time limit. The score is the number of correct answers in $90 \mathrm{~s}$, and higher DSC scores mean better functioning of processing speed. ${ }^{60}$

Attention will be measured by the Test of Attention Performance (TAP, V.2.3), which is a computer-aided standard neuropsychological test to assess attention. ${ }^{61}$ Four subtests in this test battery involving alertness for reaction, Go/Nogo, general attention and divided selective attention will be applied in this trial. ${ }^{62}$

Structure and function of related brain regions will be measured using fMRI, which is a non-invasive method for examining brain activity and structure. T1-weighted structural images are acquired with the three-dimensional magnetisation-prepared rapid acquisition gradient-echo (3DMPRAGE) sequence (164 slices; field of view (FOV) $240 \mathrm{~mm}$; slice thickness $1 \mathrm{~mm}$; inversion time $450 \mathrm{~ms}$; echo time $30 \mathrm{~ms}$; flip angle (FA) $150^{\circ}$; matrix $256 \times 256$ ). The resting state sequence will be performed (axial (nonoblique); 42 slices; FOV $200 \mathrm{~mm} ; 3 \mathrm{~mm}$ slices with $0.6 \mathrm{~mm}$ gap; repetition time $2100 \mathrm{~ms}$; echo time $30 \mathrm{~ms}$; FA $90^{\circ}$; acquisition matrix $64 \times 64 ; 3.125 \times 3.125 \mathrm{~mm}$ inplane resolution). The fMRI scan will be measured by professional operators at the Affiliated Rehabilitation 
Hospital of FJTCM using a 3.0 T signal MRI scanner (GE Healthcare, Little Chalfont, UK) with a birdcage head coil. $^{63}$

Cerebrovascular function of the vertebral, basilar, middle cerebral, anterior cerebral and posterior cerebral arterial territories of the brain, consisting of cerebrovascular haemodynamics and cerebrovascular elasticity, will be measured using a colour Doppler ultrasound imaging device (Philips; product type IU22) by professional operators at the Affiliated Rehabilitation Hospital of FJTCM.

Quality of life will be measured by the Medical Outcomes Study 36-Item Short-Form Health Survey (SF-36). ${ }^{64}$ It consists of 36 items that assess eight health concepts: functional capacity (10 items), physical activities (4 items), social activities ( 2 items), bodily pain ( 2 items), mental health (5 items), usual activities ( 4 items), vitality (4 items), and general health status (5 items). ${ }^{65}$ Each health concept is evaluated separately by the normalised scores of $0-100$, with 0 corresponding to worst health status and 100 corresponding to best health status. The Chinese version of SF-36 has been reported to have high reliability and validity in the Chinese population. ${ }^{66} 67$

\section{Safety measurements}

Any unexpected adverse events during the intervention period will be monitored, reported to a research assistant, and causality in relation to the Baduanjin or brisk walking exercise intervention and the severity of adverse events will be analysed. Serious adverse events will be reported to the ethics committee immediately.

\section{Sample size}

Sample size was calculated on the basis of improvement in MoCA scores. The mean difference and its SD of MoCA scores after the intervention between an aerobic exercise and control group are 1.826 and 0.65 points according to relevant published literature. ${ }^{68}$ We expect at least 0.3 effect size of this outcome after the 24-week Baduanjin exercise intervention. A sample size of 111 participants has been calculated to be sufficient to detect the target effect size with a type 1 error of $5 \%$ $(\alpha=0.05)$ and $80 \%$ power $(\beta=0.20)$ by Gpower 3.1.9.2 software. Considering a $20 \%$ attrition rate, a total of 135 participants is necessary, with 45 participants in each group.

\section{Statistical analysis}

Statistical analyses will be performed using the SPSS V.21.0 software package, with a statistical significance of a two-sided $\mathrm{p}$ value $<0.05$. An intention to treat (ITT) analysis, being in line with the CONSORT statement, will be used to analyse primary and secondary outcomes, and a multiple imputation method will be used to fill in missing data. Five complete datasets will be created after imputation. Each of the imputed datasets will be separately analysed, and the results of five analyses will be summarised using Rubin's rules. ${ }^{69}$ Continuous variables will be expressed as mean (SD) for normal distribution or median (IQR) for non-normal distribution, and categorical variables will be expressed as frequencies or percentages.

Baseline characteristics among the three groups will be compared using one-way analysis of variance or the Kruskal-Wallis $\mathrm{H}$ test for continuous variables and Pearson $\chi^{2}$ or Fisher's exact test for categorical variables. If incomparability appears, the inequality factors will be treated as confounding variables in the final efficacy analysis.

For the analysis of primary and secondary outcomes, the mixed-effects linear model with restricted maximum likelihood will be used to analyse the effect of the intervention on the continuous variables, and logistic regression models for dependent categorical variables. In each model, the independent variables are the group (Baduanjin group, brisk walking group and usual physical activity group), time (different measurement time points, such as baseline, post-intervention, and post-follow-up), confounding factors (eg, age, education) and any inequality factors among groups at the baseline assessment. The post hoc comparison between groups at different time points will be conducted using multiple comparisons with adjustment to the type 1 error rate.

The resting fMRI data will be analysed by independent component analysis and voxel-based morphometry to detect the strength of the correction coefficients for the brain regions related to cognitive function, which include the precuneus/posterior cingulate cortex, medial prefrontal cortex, medial temporal lobe, angular gyrus, lateral temporal cortex and medial, lateral and inferior parietal cortex and hippocampus. The multiple comparisons among the three groups will be performed using random-effects general linear model analysis. The correlation of blood oxygen level dependent (BOLD) signal changes between groups will be analysed using Pearson or Spearman linear correlations. Adverse events will be listed and analysed using a $\chi^{2}$ or Fisher's exact test, and severe adverse events will be listed in detail.

\section{Data collection and management}

The data will be collected by the outcome assessors using print-based case report forms ( $\mathrm{p}$-CRFs). Then the p-CRFs will be transcribed into web-based case report forms (w-CRFs) with an electronic data capture system (EDC) by research assistants. A data management system based on the EDC system and web servers will be provided by Centre of Data Management of FJTCM using a medical research platform (http://210.34.74.191/srtp/ users/loginlangth.action), and will meet the available standards of security. Participants' data in w-CRFs will be stored in the EDC system at separate password-protected locations. The research assistants will be responsible for ensuring the integrity and accuracy of all data when data are transcribed into w-CRFs by means of checks on value ranges and logical checks. Development and validation of the study database, quality control, and extraction of data will be performed according to the procedures of 
the medical research platform. The responsibilities $\log$ will identify all trial personnel responsible for data collection, data entry and handling, and management of the database.

\section{Ethics issues}

This protocol is conducted in accordance with the Declaration of Helsinki. Ethics approval has been obtained from the Medical Ethics Committee of the Second People's Hospital of Fujian Province (approval number 2014-KL045-02). All participants will provide voluntary written informed consent after a full discussion about the potential benefits and risks before participation.

\section{Dissemination}

The study protocol has been registered, and is available on the Chinese Trial Registry website (registered in ChiCTR.org with the identifier ChiCTR-ICR-15005795). This study will be published in scientific journals to target a wide range of groups, and presented at national conferences in the field of cognitive rehabilitation. Study results will also be sent to study participants and disseminated to researchers, healthcare providers and healthcare professionals, as well as the general public through courses, presentations and internet regardless of the magnitude or direction of effect.

\section{DISCUSSION}

An increasing number of studies have shown that an exercise (especially aerobic exercise) intervention may be beneficial for maintaining or even improving cognitive function in older adults with or without cognitive impairment. However, the optimal workload related to the type, frequency, intensity and duration of the exercise sessions has not been clearly established. ${ }^{21}$ Baduanjin exercise is an aerobic exercise characterised by simple, slow, relaxing movements. ${ }^{70}$ It consists of eight sections of movement, each of which benefits different parts of the body. ${ }^{45}$ Previous studies have indicated that Baduanjin exercise can improve sleep quality, ${ }^{71}$ cardiopulmonary function ${ }^{29}$ and blood lipid metabolism ${ }^{37}$ in community-dwelling older adults, all of which are risk factors for cognitive decline. However, there is currently a lack of evidence about the beneficial associations between Baduanjin exercise and cognitive function. This protocol describes a randomised three-arm parallel controlled trial assessing the effect of regular Baduanjin exercise on cognitive function in older adults with MCI. This trial will employ rigorous methods to reduce bias, such as randomisation, blinding of the outcome assessors and statistical analysers, samples recruited from a community-dwelling elderly population, and statistical analysis according to the ITT principle. We will also invite two qualified physical exercise teachers to serve as the Baduanjin exercise coaches to ensure standardised exercise training for the participants. A specific type of aerobic exercise (ie, Baduanjin
Qigong) with an accessible exercise scheme (60 min per day on 3 days each week for 24 weeks) will be applied as the intervention, making it possible to clearly clarify the association with cognitive function or specific domains of cognition such as memory, execution and attention. Furthermore, the extensive outcome assessment on several levels involving neuropsychological testing, restingstate fMRI and cerebrovascular function assessment will make it possible to explore the mechanism of action of the Baduanjin intervention on cognitive functional networks and brain structure, allowing the researchers to investigate the relationship between subjective scales and objective indicators. Another highlight of this trial will be the exploration of differences in effects of a mind-body exercise (Baduanjin) and a single aerobic exercise (brisk walking) on cognitive function and their possible mechanisms. In addition, the proposed study will be the first to investigate the effects of Baduanjin exercise on older adults with MCI. The results should be helpful in improving the cognitive function of the participants with MCI and should be generalisable to the community-dwelling older population.

A potential limitation of this protocol is that participants and exercise coaches cannot be blinded because it is difficult to conduct blinding in non-pharmacological trials. ${ }^{72}$ Therefore performance bias may be inevitable, but the exercise coaches will be involved in the programme of recruitment, assessment and data analysis of this study. In addition, as participants in the intervention and control groups will be recruited from the same community centre, interventional contamination from the control group will be difficult to control, although we will explain in detail the objectives and requirements of participating in this trial and record the daily activity of the participants.

In summary, this study will be the first RCT to evaluate the impact of Baduanjin exercise on cognitive function in a community-dwelling older population with MCI. The results of this trial will help to establish the optimal approach for treating cognitive decline in older adults with MCI and provide reliable evidence for its application in the rehabilitation of Traditional Chinese Medicine.

Acknowledgements This study is supported by the National Natural Science Foundation of China (http://www.nsfc.gov.cn, grant no 81574045), the Collaboration Innovation Center for Rehabilitation Technology (2015003-Collaboration), the Fujian Provincial Rehabilitation Industrial Institution and the Fujian Key Laboratory of Rehabilitation Technology. The funders had no role in the study design, data collection and analysis, decision to publish, or preparation of the manuscript.

Contributors LC, JT and GZ conceived the study, designed the study protocol, and drafted the manuscript. MH wrote the manuscript and participated in the coordination and implementation of the study. GZ wrote several sections of the manuscript and revised and finalised the study protocol. JT is in charge of coordination and direct implementation. LS, LM, $\mathrm{RX}$ and $\mathrm{ZW}$ helped to develop the study measures and analyses. All authors contributed to drafting the manuscript and have read and approved the final manuscript.

Funding Collaboration Innovation Center for Rehabilitation Technology; National Natural Science Foundation of China, 81574045.

Competing interests None declared. 
Patient consent Obtained

Ethics approval Medical Ethics Committee of the Second People's Hospital of Fujian Province (approval number 2014-KL045-02).

Provenance and peer review Not commissioned; externally peer reviewed.

Trial status Recruitment started while the manuscript was being finished.

Open Access This is an Open Access article distributed in accordance with the Creative Commons Attribution Non Commercial (CC BY-NC 4.0) license, which permits others to distribute, remix, adapt, build upon this work noncommercially, and license their derivative works on different terms, provided the original work is properly cited and the use is non-commercial. See: http:// creativecommons.org/licenses/by-nc/4.0/

\section{REFERENCES}

1. [No authors listed]. A global assessment of dementia, now and in the future. Lancet 2015;386:931.

2. Seitz D, Purandare N, Conn D. Prevalence of psychiatric disorders among older adults in long time care homes: a systematic review. Int Psychogeriatr 2010;22:1025-39.

3. Chan WC, Lam LC, Tam CW, et al. Neuropsychiatric symptoms are associated with increased risk of progression to dementia: a 2-year prospective study of 321 Chinese older persons with mild cognitive impairment. Age Ageing 2011;40:30-5.

4. Giebel CM, Sutcliffe C, Challis D. Activities of daily living and quality of life across different stages of dementia: a UK study. Aging Ment Health 2015;19:63-71.

5. Flak MM, Hernes SS, Skranes J, et al. The Memory Aid study: protocol for a randomized controlled clinical trial evaluating the effect of computer-based working memory training in elderly patients with mild cognitive impairment (MCl). Trials 2014;15:156.

6. Farias ST, Mungas D, Reed BR, et al. Progression of mild cognitive impairment to dementia in clinic- vs community-based cohorts. Arch Neurol. 2009;66:1151-7.

7. Lyketesos CG, Lopez O, Jones B, et al. Prevalence of neuropsychiatric symptoms in dementia and mild cognitive impairment: results from the cardiovascular health study. JAMA 2002:288:1475-83.

8. Alzheimer's Association. 2009 Alzheimer's disease facts and figures Alzheimers Dement 2009;5:234-70.

9. Larrieu S, Letenneur L, Orgogozo JM, et al. Incidence and outcome of mild cognitive impairment in a population-based prospective cohort. Neurology 2002;59:1594-9.

10. Loewenstein DA, Acevedo A, Small BJ, et al. Stability of different subtypes of mild cognitive impairment among the elderly over a 2 to 3-year follow-up period. Dement Geriatr Cogn Disord 2009;27:418-23.

11. Gao S, Unverzagt FW, Hall KS, et al. Mild cognitive impairment, incidence, progression, and reversion: findings from a community-based cohort of elderly African Americans. Am J Geriatr Psychiatry 2014;22:670-81.

12. Levin OS, Yunishchenko NA, Dudarova MA. Efficacy of akatinol memantine in moderate cognitive impairments. Neurosci Behav Physiol 2010;40:926-33.

13. Hamer M, Chida Y. Physical activity and risk of neurodegenerative disease: a systematic review of prospective evidence. Psychol Med 2009;39:3-11.

14. Ahlskog JE, Geda YE, Graff-Radford NR, et al. Physical exercise as a preventive or disease-modifying treatment of dementia and brain aging. Mayo Clin Proc 2011;86:876-84.

15. Baker LD, Frank LL, Foster-Schubert K, et al. Effects of aerobic exercise on mild cognitive impairment. Arch Neurol 2010;67:71-9.

16. Grande G, Vanacore N, Maggiore L, et al. Physical activity reduces the risk of dementia in mild cognitive impairment subjects: a cohort study. J Alzheimers Dis 2014;39:833-9.

17. Smith PJ, Blumenthal JA, Hoffman BM, et al. Aerobic exercise and neurocognitive performance: a meta-analytic review of randomized controlled trials. Psychosom Med 2010;72:239-52.

18. Tanigawa $\mathrm{T}$, Takechi $\mathrm{H}$, Arai $\mathrm{H}$, et al. Effect of physical activity on memory function in older adults with mild Alzheimer's disease and mild cognitive impairment. Geriatr Gerontol Int 2014;14:758-62.

19. Middleton LE, Barnes DE, Lui LY, et al. Physical activity over the life course and its association with cognitive performance and impairment in old age. J Am Geriatr Soc 2010;58:1322-6.

20. Hillman $\mathrm{CH}$, Erickson $\mathrm{KI}$, Kramer AF. Be smart, exercise your heart: exercise effects on brain and cognition. Nat Rev Neurosci 2008;9:58-65.
21. Paillard T. Preventive effects of regular physical exercise against cognitive decline and the risk of dementia with age advancement. Sports Med Open 2015;1:4.

22. Carvalho A, Rea IM, Parimon T, et al. Physical activity and cognitive function in individuals over 60 years of age: a systematic review. Clin Interv Aging 2014;9:661-82.

23. Kirk-Sanchez NJ, McGough EL. Physical exercise and cognitive performance in the elderly: current perspectives. Clin Interv Aging 2014;9:51-62.

24. Snowden M, Steinman L, Mochan K, et al. Effect of exercise on cognitive performance in community-dwelling older adults: review of intervention trials and recommendations for public health practice and research. J Am Geriatr Soc 2011;59:704-16.

25. Lautenschlager NT, Cox KL. Can participation in mental and physical activity protect cognition in old age? Comment on "The mental activity and exercise (MAX) trial: a randomized controlled tria to enhance cognitive function in older adults". JAMA Intern Med 2013;173:805-6.

26. Li R, Jin L, Hong P, et al. The effect of baduanjin on promoting the physical fitness and health of adults. Evid Based Complement Alternat Med 2014:2014:784059.

27. Koh TC. Baduanjin-an ancient Chinese exercise. Am J Chin Med 1982;10:14-21.

28. An B, Dai K, Zhu Z, et al. Baduanjin alleviates the symptoms of knee osteoarthritis. J Altern Complement Med 2008;2:167-74.

29. Xue WS. The effect of healthy Qigong-Baduanjin on cardiopulmonary function in elder adults. $J$ Bohai Univ 2013:34:431-5.

30. Zhai FM, Chen YJ, Huang ZF, et al. Effect of Baduanjin exercise on physiological function in old people. Chin J Gerontol 2013:33:1402-5.

31. Zeng YG, Zhou XQ, Wang AL, et al. Research on the impacts of fitness Qigong-Baduanjin on figure and physical function among the middle-aged and aged people. J Beijing Univ Phys Educ 2005;28:1207-9.

32. An BC, Wang $Y$, Jiang $X$, et al. Effects of Baduanjin exercise on knee osteoarthritis: a one-year study. Chin J Integr Med 2013;19:143-8.

33. Chen HH, Yeh ML, Lee FY. The effects of Baduanjin qigong in the prevention of bone loss for middle-aged women. Am J Chin Med 2006;34:741-7.

34. Wang ST. Effect of Baduanjin on physiological age of intelligence for old people. J Clin Rehabil Tissue Eng Res 2007;11:7910-13.

35. Sun G, Wang AL. The influences of two different exercise means on the intellectual physiological age of the Male elderly people. $J$ Beijing Univ Phys Educ 2008;31:1378-80.

36. Xiong X, Wang P, Li S, et al. Effect of Baduanjin exercise for hypertension: a systematic review and meta-analysis of randomized controlled trials. Maturitas 2015;80:370-8.

37. Mei L, Chen $\mathrm{Q}, \mathrm{Ge} \mathrm{L}$, et al. Systematic review of Chinese traditional exercise baduanjin modulating the blood lipid metabolism. Evid Based Complement Alternat Med 2012;2012:282131.

38. Huan T, Chang JD. Influences of body building Qigong-Baduanjin on the metabolisms of nitric oxide, malondialdehyde and superoxide dismutase in middle-aged and elderly people of different gender. Chin J Clin Rehabil 2005;9:162-4.

39. Liao $\mathrm{Y}$, Lin $\mathrm{Y}$, Zhang $\mathrm{C}$, et al. Intervention effect of Baduanjin exercise on the fatigue state in people with fatigue-predominant subhealth: a cohort study. J Altern Coplement Med 2015;21:554-62.

40. Cheng FK. Effects of Baduanjin on mental health: a comprehensive review. J Bodyw Mov Ther 2015;19:138-49.

41. Chan JSM, Ho RTH, Chung K, et al. Qigong exercise alleviates fatigue, anxiety, and depressive symptoms, improves sleep quality, and shortens sleep latency in persons with chronic fatigue syndrome-like illness. Evid Based Complement Alternat Med 2014;2014:106048.

42. Chan AW, Tetzlaff JM, Altman DG, et al. SPIRIT 2013 statement: defining standard protocol items for clinical trials. Ann Intern Med 2013;158:200-7.

43. Schulz KF, Altman DG, Moher D. CONSORT 2010 statement: updated guidelines for reporting parallel group randomised trials. Ann Intern Med 2010;4:291-2.

44. Peterson RC. Mild cognitive impairment as a diagnostic entity. $J$ Intern Med 2004;256:183-94.

45. Health Qigong Management Center of General Administration of Sport of China: Health Qigong-Baduanjin. Beijing: People's Sports Publishing House of China, 2003.

46. Zheng GH, Li MY, Lan XL, et al. The effect of Baduanjin exercise for physical and psychological wellbeing of college students: 
study protocol for a randomized controlled trial. Trials 2013;14:422.

47. Ying F. An experimental study on the effects of brisk walking upon cardiovascular function of 60-69 year old people [D]. Shandong Institute of P.E. and Sports, 2012.

48. Nasreddine ZS, Phillips NA, Bédirian V, et al. The Montreal Cognitive Assessment, MoCA: a brief screening tool for mild cognitive impairment. J Am Geriatr Soc 2005;53:695-9.

49. Yusupu N, Yi Z. An introduction of Montreal Cognitive Assessment (MoCA) and a comparison among its versions. J Neurosci Mental Health 2010;10:516-19.

50. Gong-wei J, Qi S, Ying Y, et al. A preliminary study of application of montreal cognitive assessment in Chongqing city. Neural Injury Funct Reconstruction 2008;3:41-2.

51. Reitan RM, Wolfson D. The halstead-reitan neuropsychological test battery. Tuscon: Neuropsychology Press, 1985.

52. Arbuthnott K, Frank J. Trail marking test, part B as a measure of executive control: validation using a set-switching paradigm. J Clin Exp Neuropsychol 2000;22:518-28.

53. Morooka T, Ogino T, Takeuchi A, et al. Relationships between the color-word matching Stroop task and the Go/NoGo task: toward multifaceted assessment of attention and inhibition abilities of children. Acta Med Okayama 2012;66:377-86.

54. Dong G, Zhou H, Zhao X. Male internet addicts show impaired executive control ability: evidence from a color-word Stroop task. Neurosci Lett 2011;499:114-18.

55. Li L, Shi-jie Z. The application of Wechsler memory scale revised in China in Chinese children. Chin J Clin Psychol 2009;17:705-7.

56. Yaoxian G. The handbook of Wechsler adult intelligence scale-revised in Chinese. Hunan Medical University, 1989:33-48.

57. Xie D. The neuropsychological study on false memory in patients with amnesia mild cognitive impairment[D]. Anhui Medical University, 2011.

58. Hong $X$, Zhang ZX, Wu LY, et al. Validity of auditory verbal learning test in diagnosis of Alzheimer's disease. Zhongguo Yi Xue Ke Xue Yuan Xue Bao 2012;34:262-6.

59. Qi-hao G, Yi-min S, Pei-min Y, et al. Norm of auditory verbal learning test in the normal aged in China community. Chin J Clin Psychol 2007;15:132-41.
60. Yaoxian G. The revision of Wechsler adult intelligence scale. Act Psychological Sinicae 1983;15:362-9.61.

61. Test of Attentional Performance (TAP, Version 2.3). Psytest. http:// www.psytest.net (accessed 13 Oct 2015)

62. Beck L, Heusinger A, Boecker M, et al. Convergent and predictive validity of two computerized attention tests in brain-damaged patients. Zeitschrift für Neuropsychologie 2008;19:213-22.

63. Lang S, Duncan N, Northoff G. Resting-state functional magnetic resonance imaging: review of neurosurgical applications. Neurosurgery 2014;74:453-64.

64. Qu B, Guo HQ, Liu J, et al. Reliability and validity testing of the SF-36 questionnaire for the evaluation of the quality of life of Chinese urban construction workers. J Int Med Res 2009;37:1184-90.

65. Ware JE, Sherbourne CD. The Mos 36-item short-form health survey (SF-36). Med Care 1992;30:473-83.

66. Jin WZ, Yu HT. A study of the reliability and validity of SF-36 scale on evaluating health of population. Chin Health Resour 2012;15:265-7.

67. Lam CL, Tse EY, Gandek B, et al. The sf-36 summary scales were valid, reliable, and equivalent in a Chinese population. $J$ Clin Epidemiol 2005;58:815-22.

68. Nascimento CM, Pereira JR, Pires de Andrade L, et al. Physical exercise improves peripheral BDNF levels and cognitive functions in elderly mild cognitive impairment individuals with different BDNF Val66Met Genotypes. J Alzheimers Dis 2015;43:81-91.

69. Schafer JL. Multiple imputation: a primer. Stat Methods Med Res 1999:8:3-15.

70. Lin Q. Baduanjin and Chinese medical health preservation. J Fujian Univ Tradit Chin Med 2010;20:55-6.

71. Chen MC, Liu HE, Huang HY, et al. The effect of a simple traditional exercise programme (Baduanjin exercise) on sleep quality of older adults: a randomized controlled trial. Int J Nurs Stud 2012;49:265-73.

72. Boutron I, Moher D, Altman DG, et al., CONSORT Group. Extending the CONSORT statement to randomized trials of nonpharmacologic treatment: explanation and elaboration. Ann Intern Med 2008;148:295-309. 